\title{
The association between WNT10A variants and dental development in patients with isolated oligodontia
}

\author{
Brunilda Dhamo $^{1,2}$, Willem Fennis ${ }^{3}$, Marijn Créton ${ }^{3}$, Strahinja Vucic ${ }^{1,2}$, Marco Cune ${ }^{4,5}$, \\ Hans Kristian Ploos van Amstel ${ }^{6}$, Eppo B Wolvius ${ }^{1,2}$, Marie-José van den Boogaard ${ }^{6}$ and \\ Edwin M Ongkosuwito ${ }^{\star, 1,2}$
}

In this study we aimed to determine the effect of WNT10A variants on dental development in patients with oligodontia. Fortythree ( 25 boys and 18 girls) individuals were eligible for this study. Stage of development for each present tooth was assessed using the Demirjian method. In case no corresponding tooth was present, regression equations were applied for dental age to be calculated. The ratio between length of root and length of crown was ascertained for each present tooth in all quadrants. All patients were physically examined by a clinical geneticist and DNA analysis of the WNT10A gene was performed. Linear regression models were applied to analyze the association between WNT10A variants and dental age. The same analysis was applied to study the association between WNT10A variants and root elongation for each present tooth. One ordinal regression model was applied to analyze the association between WNT10A variants and development of present maxillary and mandibular teeth. Thirty-six (84\%) patients were detected with WNT10A variants of which six patients displayed evident ectodermal features. Dental age was $1.50(95 \%$ confidence interval $(\mathrm{Cl}):-2.59,-0.42)$ to $1.96(95 \% \mathrm{Cl}:-3.76,-0.17)$ years lower in patients with WNT10A variants compared with patients without variants. The development of maxillary canine, maxillary second molar and mandibular second molar was statistically significantly delayed in patients with WNT10A variants compared with patients without variants. The impact of WNT10A variants on dental development increases with presence of the nonsense c. (321C $>$ A p. $\left(\mathrm{C}_{107^{*}}\right)$ ) variant and the number of missing teeth.

European Journal of Human Genetics (2017) 25, 59-65; doi:10.1038/ejhg.2016.117; published online 21 September 2016

\section{INTRODUCTION}

Dental agenesis is defined as the congenital absence of one or more primary or secondary teeth, excluding the third molars. ${ }^{1,2}$ As a frequent dental anomaly, ${ }^{3}$ dental agenesis in the permanent dentition is studied for its prevalence in different populations. ${ }^{4-6}$ The prevalence of dental agenesis in the Dutch population is estimated to be $5 \%$, similar to the prevalence in Europe. ${ }^{4,7}$ Dental agenesis can be classified as hypodontia (1-5 missing teeth), oligodontia ( $\geq 6$ missing teeth) or anodontia (no tooth present). ${ }^{2,8,9}$ Oligodontia is observed in $\sim 0.14 \%$ of the population ${ }^{10}$ and specifically for the Dutch population the prevalence of isolated oligodontia is reported to be $0.08 \% .{ }^{11}$ The numeric distinction between hypodontia, oligodontia and anodontia seems an arbitrary one, as the location of missing teeth is important as well. ${ }^{12}$ Due to the lack of teeth, oligodontia has an impact on the quality of life. ${ }^{13}$ Furthermore, dental agenesis is associated with a delay of dental development, abnormal size and abnormal shape of teeth. ${ }^{14-19}$ Dental agenesis is a hereditary condition with both environmental and genetic factors as part of etiology. ${ }^{10,20}$ Genetic evidence of dental agenesis has been previously presented. ${ }^{21-26}$ MSX1, PAX9, AXIN2, EDA, EDAR, EDARADD, LRP6 and WNT10A variants in mice are associated with dental agenesis and an aberrant development of the dentition. ${ }^{27-36}$ Recent findings associate compound heterozygosity/homozygosity or heterozygosity for WNT10A variants with isolated dental agenesis in humans. ${ }^{7,37}$ In addition WNT10A variants are associated with various ectodermal dysplasias (EDs; OMIM, Entry 305100) often corresponding to the odontoonychodermal dysplasia (OODD; OMIM, Entry 257980) and Schöpf-Schulz-Passarge syndrome (SSPS; OMIM, Entry 224750), which combine abnormal development of ectodermal structures including teeth. ${ }^{38-41}$ Van den Boogaard et $a l^{7}$ showed that variants of WNT10A were present in more than half cases of isolated oligodontia $v s<10 \%$ variants of MSX1, PAX9 and AXIN2, concluding that the normal expression of the WNT10A gene is important for the formation of the tooth germ. However less information is available on the association between WNT10A variants and dental development. As WNT10A is strongly expressed in the dental epithelium at the initiation stage and has a role in tooth development beyond the bud stage, one may hypothesize that delayed dental development is part of the phenotype WNT10A dental agenesis. ${ }^{32,33}$ More insight into genotype-phenotype association facilitates the recognition of a possible genetic etiology in patients with dental agenesis, which may have consequences for the treatment approach. The aim of this study was to determine the effect of WNT10A variants on dental development in patients with oligodontia.

\footnotetext{
${ }^{1}$ Department of Oral \& Maxillofacial Surgery, Special Dental Care and Orthodontics, Erasmus University Medical Centre, Rotterdam, The Netherlands; ${ }^{2}$ The Generation R Study Group, Department of Epidemiology, Erasmus University Medical Centre, Rotterdam, The Netherlands; ${ }^{3}$ Department of Oral and Maxillofacial Surgery, Prosthodontics and Special Dental Care, University Medical Centre Utrecht, Utrecht, The Netherlands; ${ }^{4}$ Department of Oral Function and Prosthodontics, UMC Groningen, Groningen, The Netherlands; ${ }^{5}$ Centre for Special Dental Care, St Antonius Hospital, Nieuwegein, The Netherlands; ${ }^{6}$ Department of Medical Genetics, University Medical Center Utrecht, Utrecht, The Netherlands

${ }^{*}$ Correspondence: Dr EM Ongkosuwito, Department of Oral \& Maxillofacial Surgery, Special Dental Care and Orthodontics, Erasmus University Medical Centre, PO Box 2040, 3000 Rotterdam, The Netherlands. Tel: +3110 7036426; Fax: +3110 7036810; E-mail: e.ongkosuwito@erasmusmc.nl

Received 21 January 2016; revised 25 July 2016; accepted 5 August 2016; published online 21 September 2016
} 


\section{MATERIALS AND METHODS}

\section{Study population}

Individuals with oligodontia visiting the Departments of Oral and Maxillofacial Surgery, Prosthodontics and Special Dental Care of the University Medical Center Utrecht (UMC Utrecht) and the St Antonius Hospital, Nieuwegein, The Netherlands were referred to the Department of Medical Genetics of the UMC Utrecht for syndrome diagnostics and genetic counseling. Informed consents were obtained from the patients or parents. The data of this study (patient IDs;73681-73716) including variants and phenotypes were submitted into LOVD-funded database (http://databases.lovd.nl/shared/ variants/0000117595). Forty-three ( 25 boys and 18 girls) patients with a mean age of 10.77 (SD 2.4) years were eligible for this study according to the following inclusion criteria: oligodontia (six or more missing teeth, excluding third molars), age between 6 and 16 years old and the presence of a dental panoramic radiograph (DPR). Mean age of the boys was 11.05 (SD 2.59) years and mean age of the girls was 10.38 years (SD 2.16) (Table 1).

\section{Oligodontia assessment}

Oligodontia was assessed by clinical examination from the dentist and also by DPRs. A tooth was classified as missing when no sign of formation or calcification showed in the DPR. Patterns of oligodontia were identified using the tooth agenesis code (TAC). The TAC is a unique number that is consistent with a specific pattern of dental agenesis. ${ }^{42,43}$

\section{Dental development assessment}

Dental development was defined using the Demirjian method. ${ }^{44}$ One experienced examiner determined one of the eight developmental stages (A, B, C, D, E, F, G and H) for each present tooth in all the quadrants.

Dental age was calculated for each patient referring to the stages of development of teeth in the left quadrant as follows. In order to estimate the stage of development for the missing teeth, a combined method was applied. This method consists of assessing the stage of development for a missing tooth in the lower left quadrant from the corresponding right mandibular tooth or from a corresponding maxillary tooth if the tooth was missing in both sides of the mandible. In case no corresponding tooth was present, regression equations developed by Nystrom et al $l^{45}$ were applied. These equations take into account the development of the remaining teeth in the lower left quadrant, age and sex of the patient to calculate dental age. Obtained stages of dental development were used to calculate the dental maturity score by summing up the weighted scores given to every tooth of the lower left quadrant. ${ }^{46}$ Finally, the Dutch dental age standard tables for boys and girls were used to convert the dental maturity score into dental age. ${ }^{46}$
To obtain a better approach of dental development in our patients, additional measurements were performed using DPRs. In order to estimate the root elongation, the examiner ascertained the ratio between length of root and length of crown for each present tooth in all quadrants. Abnormal shape or size of teeth and permanent dental fillings were signed when detected.

\section{Physical examination}

All patients were physically examined by a single clinical geneticist, in order to identify possible features of ED (skin, hair, nails and sweat glands) or other syndromes. In addition, deviations in function of sweat glands, skin, hair and nails were assessed using a standardized form. The patients were classified as displaying evident ectodermal features or not.

\section{Detection and analysis of WNT10A variants}

Blood samples were obtained and DNA analysis of the WNT10A and the MSX1, PAX9, AXIN2 genes was performed essentially, as described in a previous study. ${ }^{7}$ High-molecular weight genomic DNA was extracted from blood samples using standard procedures. PCR amplification of all exons of WNT10A and their splice site consensus sequences was applied. Mutation analysis was performed using the genetic analysis software Sequence Pilot V. 3.4.4 (JSI Medical Systems GmbH, Kippenheim, Germany), and mutation interpretation software Alamut (Interactive Biosoftware, Rouen, France) was used for further interpretation. Nomenclature is according HGVS guidelines. In case ectodermal dysplasia was suspected, additional DNA analysis was performed for ED1, EDAR and EDARADD. To evaluate the effect of the most frequent nonsense c. $\left(321 \mathrm{C}>\mathrm{A}\right.$ p. $\left.\left(\mathrm{C} 107^{\star}\right)\right)$ variant (without gene product) and missense c. $(682 \mathrm{~T}>\mathrm{A}$ p.(F228I)) variant, the WNT10A variants were sub classified as without any variant, heterozygous/homozygous missense c.(682T >A p.(F228I)) variant, heterozygous/homozygous miscellaneous variant or heterozygous/homozygous nonsense c. $\left(321 \mathrm{C}>\mathrm{A}\right.$ p. $\left.\left(\mathrm{C} 107^{*}\right)\right)$ variant (Supplementary Table S2). The description of the WNT10A variants is presented using the accession number NM_025216.2 of the reference sequence (Supplementary Table S2).

\section{Statistical analysis}

The association between WNT10A and dental age in patients with isolated oligodontia was studied using two linear regression analysis (Table 2). The first linear regression analysis tested the association between the presence of any WNT10A variant and dental age. The second linear regression analysis tested the association between the presence of a nonsense WNT10A variant and dental age. Both linear regression analysis were performed in two consecutive models. The first model was adjusted for age and gender. The second model was

Table 1 The descriptive characteristics of patients included in the study $(N=43)$

\begin{tabular}{|c|c|c|c|}
\hline & Without WNT1OA variants $(\mathrm{No}=7)$ & With WNT1OA variants $(\mathrm{No}=36)$ & P-value \\
\hline Age (mean, SD) & $11.27(1.81)$ & $10.67(2.53)$ & 0.55 \\
\hline Dental age (mean, SD) & $10.26(1.87)$ & $7.63(2.57)$ & $0.01^{\mathrm{a}}$ \\
\hline Gender (N, \%) & & & 0.10 \\
\hline Girls & $5(11.6 \%)$ & $13(30.2 \%)$ & \\
\hline Boys & $2(4.6 \%)$ & $23(53.5 \%)$ & \\
\hline Number of missing teeth (median, $70 \%$ range) & $9(6,19)$ & $13(6,22)$ & 0.35 \\
\hline Abnormal size or shape (N, \%) & & & 0.54 \\
\hline Yes & $1(2.3 \%)$ & $8(18.6 \%)$ & \\
\hline No & $6(14.0 \%)$ & $28(65.1 \%)$ & \\
\hline Filled teeth (mean, SD) & $0.29(0.76)$ & $0.56(0.99)$ & 0.50 \\
\hline Ectodermal features (N, \%) & & & 0.69 \\
\hline Yes & $1(2.3 \%)$ & $6(14.0 \%)$ & \\
\hline No & $5(11.6 \%)$ & $30(69.8 \%)$ & \\
\hline
\end{tabular}

Abbreviation: $N$, number of participants.

${ }^{a}$ Differences were tested using independent $t$-test for continuous variables and $\chi^{2}$-test for categorical variables.

Dental age was calculated if both matching mandibular teeth were missing by scoring them as a developmental stage of the left or right matching maxillary tooth. If corresponding maxillary teeth were missing the developmental stage was calculated from regression equations developed from Nyström et al. ${ }^{45}$ 


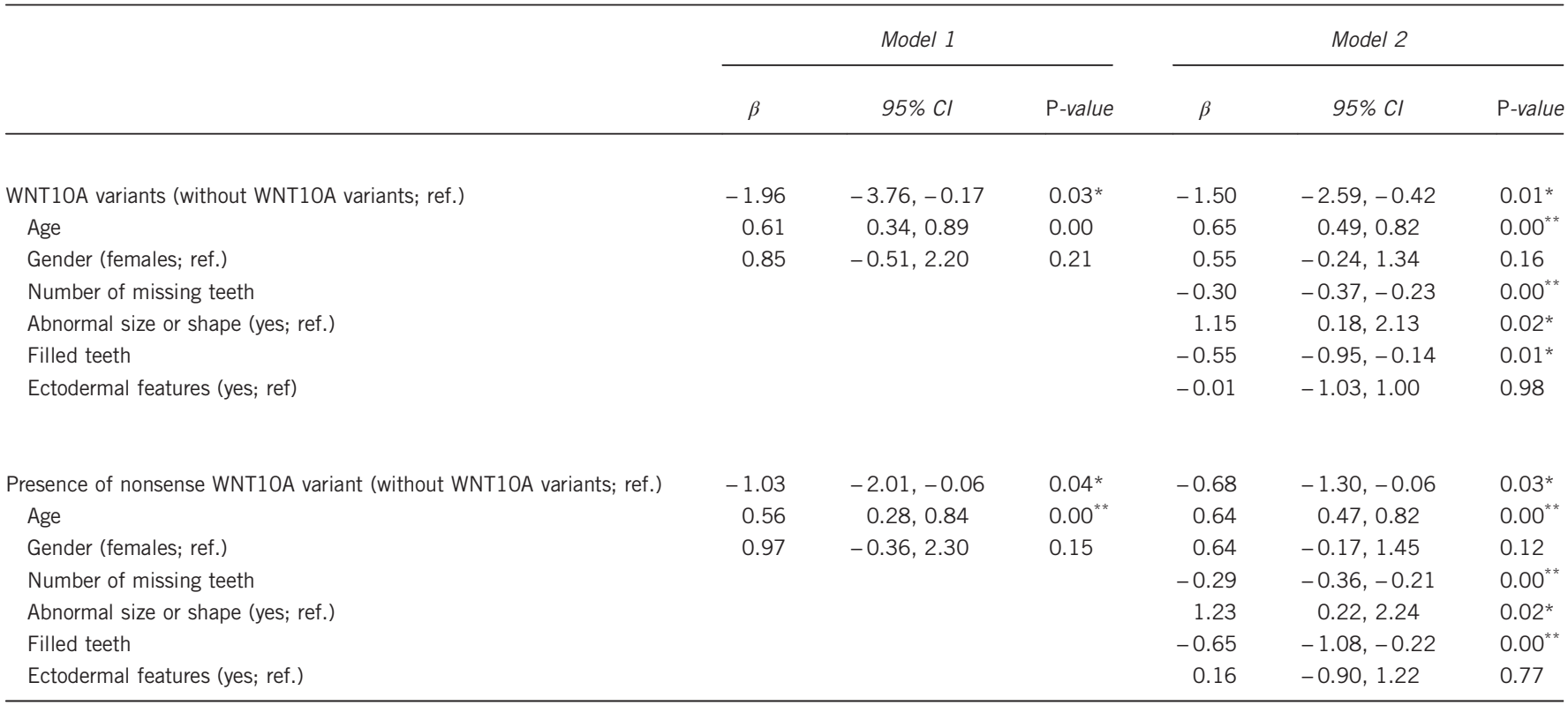

Abbreviations: $\beta$, regression coefficients, $\mathrm{Cl}$, confidence interval, ref., reference.

* Statistically significant $P<0.05$; ** Statistically highly significant $P<0.01$. Dental age was calculated if both matching mandibular teeth were missing by scoring them as follows: (a) as a developmental stage calculated from regression equations developed by Nyström et al, ${ }^{45}$ (b) as a developmental stage of the (left) matching maxillary tooth.

Model 1 was the association between WNT10A variants and dental age is adjusted for age and gender.

Model 2 was additionally adjusted for the number of missing teeth, abnormal size or shape, number of filled teeth and the presence of ectodermal features.

The presence of nonsense WNT10A variants was classified as 0 - without WNT10A variants; 1 - missense and miscellaneous WNT10A variants; 2 - nonsense WNT10A variants.

additionally adjusted for number of missing teeth, abnormal size or shape, number of filled teeth and the presence of ectodermal features.

The association between WNT10A and root elongation (length of root/ length of crown ratio) of each present tooth in dentition was analyzed using two linear regression models. The first model was adjusted for age and gender. The second model was additionally adjusted for the number of missing teeth, abnormal size or shape, number of filled teeth and the presence of ectodermal features (Table 3).

The association between WNT10A and development of the present maxillary and mandibular teeth was analyzed using one ordinal regression model adjusted for age, gender, number of missing teeth, abnormal size or shape, number of filled teeth and the presence of ectodermal features (Figure 1).

Interaction terms between gender, number of missing teeth, abnormal shape or size, presence of evident ectodermal features and WNT10A in relation with the dental development were tested. As no significant interaction terms were found, no stratification for these interaction terms was applied during analysis. All statistical analysis were performed using statistical software SPSS version 21.0 (SPSS Inc., Chicago, IL, USA).

\section{RESULTS}

Thirty-six patients (84\%) were detected with WNT10A variants (Supplementary Table S2). Of these, 11 had a missense variant (one patient showed ectodermal features), 10 had a miscellaneous variant (one patient showed ectodermal features) and 15 had a nonsense variant (four patients showed ectodermal features). Additional DNA analysis of the patients with WNT10A variants revealed no variants in the MSX1, PAX9, AXIN2 or ED1, EDAR and EDARADD genes. Of the seven patients without WNT10A variants only one showed evident ectodermal features. Additional DNA analysis of the patients without WNT10A variants revealed a PAX9 variant for one patient. There was no age and gender difference between the group of patients with WNT10A variants and the group without WNT10A variants (Table 1). There was no difference for abnormal tooth size and shape, number of missing teeth or the presence of evident ectodermal features between both the groups.

\section{Patterns of oligodontia}

The percentages of missing teeth per tooth type were similar to those from other studies. ${ }^{4,43}$ The lower second premolar (80.3\%), the upper lateral incisor $(64.0 \%)$, the lower central incisor $(64.0 \%)$ and the lower second molar (64\%) were most frequently absent. The upper central incisor was present in all the patients. The upper first molar (10.5\%) and the lower first molar (9.3\%) were less frequently absent. Patients with WNT10A variants showed a higher number of missing teeth. Furthermore, the number of missing teeth increased with the presence of a nonsense WNT10A variant $(P$ 0.001). The tooth agenesis codes were calculated per quadrant (Supplementary Table S1). The most severe oligodontia patterns were more prevalent in boys, whereas the mildest patterns were more prevalent in girls. The most frequent oligodontia patterns were more prevalent in patients with WNT10A variants than in patients without $\mathrm{WNT} 10 \mathrm{~A}$ variants.

\section{WNT10A variants and dental development}

WNT10A variants and dental age. First linear regression analysis revealed a statistically significant lower dental age of $1.50-1.96$ years for patients with WNT10A variants compared with patients without WNT10A variants (Table 2). Second linear regression analysis revealed that dental age decreased statistically significantly $0.68-1.03$ years with the presence of a nonsense WNT10A variant.

WNT10A variants and root elongation (length of root/length of crown ratio). Model 1 in the linear regression analysis showed that the roots of the left mandibular second molar were statistically significantly shorter $(\beta=-0.72$; 95\% (confidence interval) CI: $-1.23,-0.21)$ in patients with WNT10A variants compared with patients without WNT10A variants (Table 3 ). The effect estimates obtained from 
Table 3 The association between WNT10A variants and root elongation (length of root/length of crown ratio) for each present tooth

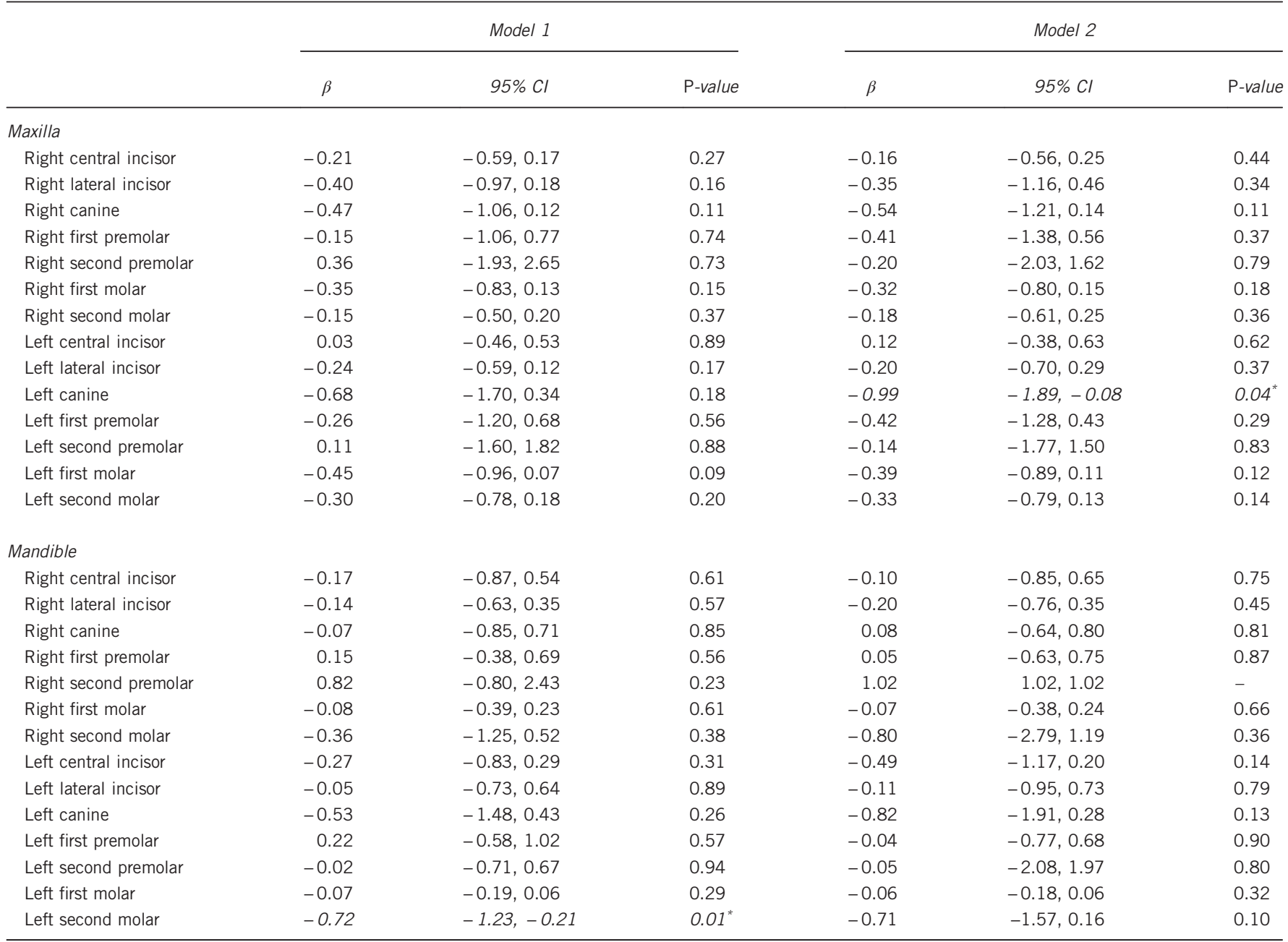

Abbreviations: $\beta$, regression coefficients, $\mathrm{Cl}$, confidence interval. * Indicates statistical significant result.

$P$-values presented from independent $t$-test for continuous variables and $\chi^{2}$-test for categorical variables. Model 1 was the association between WNT10A variants and root elongation is adjusted for age and gender. Model 2 was additionally adjusted for the number of missing teeth, abnormal size or shape, number of filled teeth and the presence of ectodermal features. The significant effect estimates and their corresponding confidence interval are presented in italic entries.

Model 1 for all the other present teeth in the dentition were not statistically significant. Model 2 in the linear regression analysis showed that the root of the left maxillary canine was statistically significantly shorter $(\beta=-0.99$; $95 \% \mathrm{CI}:-1.89,-0.08)$ in patients with WNT10A variants compared with patients without WNT10A variants. The effect estimates obtained from the fully adjusted model (Model 2) for all the other present teeth in the dentition were not statistically significant.

WNT10A variants and development of present teeth. Ordinal regression analysis revealed a statistically significant delay of 6.28 (95\% CI: $-7.58,-1.05)$ stages of development for the maxillary canine, 5.09 (95\% CI: $-8.70,-1.48)$ stages of development for the maxillary second molar and 5.21 (95\% CI: - 9.41, - 1.48) stages of development for the mandibular second molar (Figure 1). No statistically significant result was obtained for the mandibular canine, maxillary first premolar, mandibular first premolar, maxillary second premolar and mandibular second premolar. The ordinal regression analysis was performed as well for central incisors, lateral incisors and first molars in both jaws, but as these teeth were in the final stage of their development and the sample size was small, the values fell in one category and presented uninterpretable parameter estimates.

\section{DISCUSSION}

Although knowledge on the genetic cause of dental agenesis becomes available, a classification of patients can be made based on genetic characteristics. Before, patients with dental agenesis could only be classified by the number of missing teeth or as a feature of a syndrome. ${ }^{11,47}$ With information on the genotype of patients with dental agenesis, information on the phenotype facilitates an individualized patient-centered treatment approach for patients with oligodontia. One of the clinical characteristics can be a delayed dental development. Previous studies on dental agenesis and delayed dental development did not include genetic analysis. ${ }^{15-18}$ Possibly, the delayed dental development is related to the genetic etiology of the dental agenesis and caused by WNT10A. The population in the present study was relatively small. Oligodontia, however, can be considered a rare congenital dental anomaly. Furthermore, WNT10A was only recently identified as a major gene in the etiology of dental agenesis. ${ }^{7,48}$ To our knowledge a comparable group of patients with information on the dental and genetic characteristics is not available.

The aim of this study was to determine the effect of WNT10A variants on dental development in patients with oligodontia. 


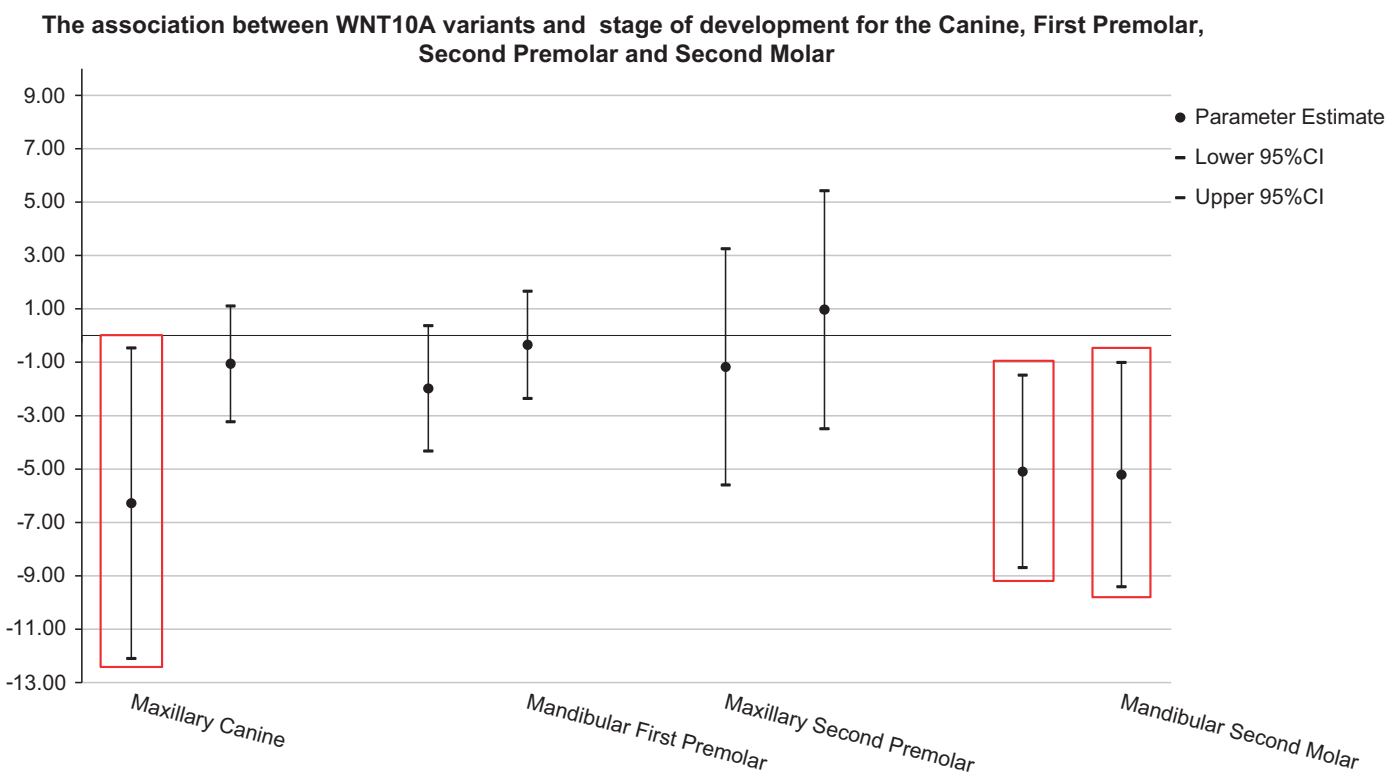

Figure 1 Box plot from ordinal regression analysis. The association between WNT10A variants and stage of development for the canine, first premolar, second premolar and second molar. • parameter estimate (lower 95\% $\mathrm{Cl}$, upper $95 \% \mathrm{Cl}$ ). The ordinal regression model was fully adjusted for age, gender, number of missing teeth, abnormal size or shape, number of filled teeth and the presence of ectodermal features; Significant parameter estimates $(P$-value $<0.05)$ are presented inside the rectangle-shaped box.

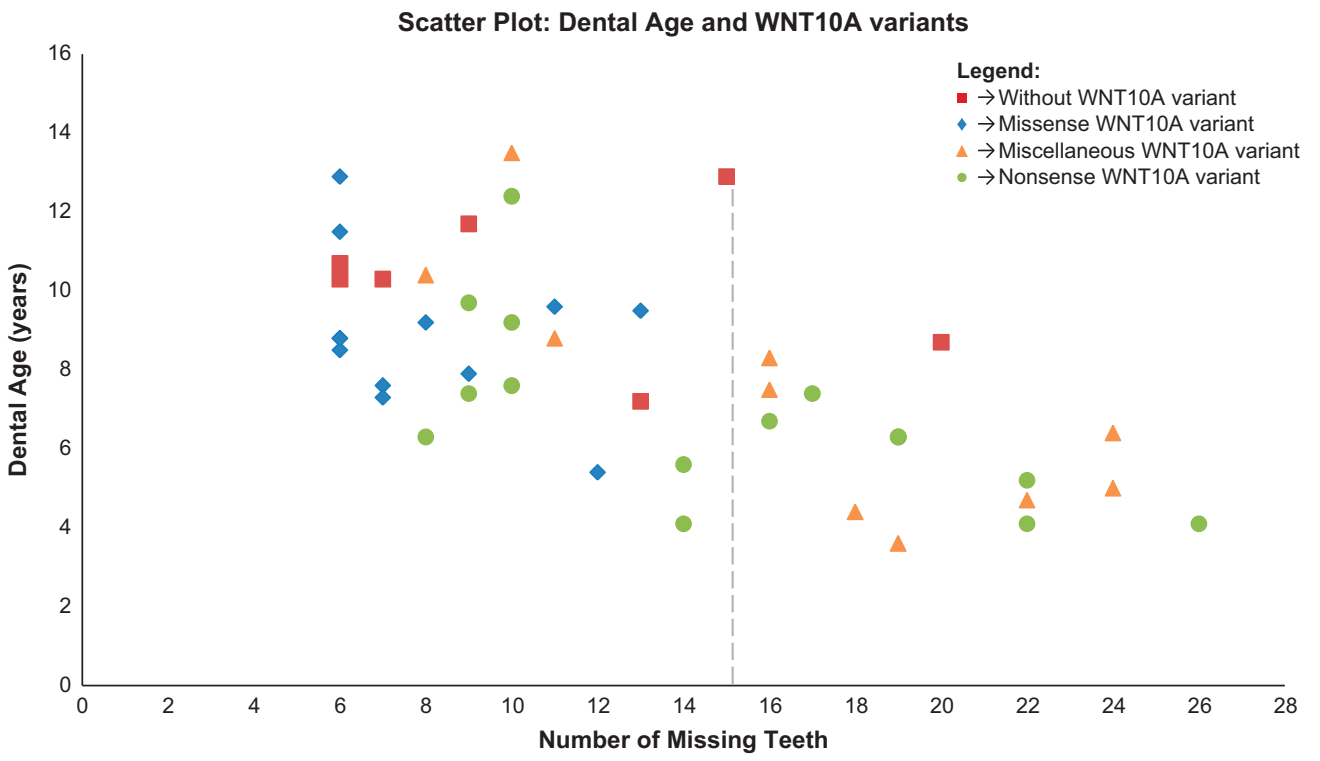

Figure 2 Schematic presentation of the association between WNT10A variants and dental age in relation to the number of the missing teeth. $\square$ without WNT10A variant. Heterozygous/homozygous P (F228I) missense variant. Heterozygous/homozygous miscellaneous variant. Heterozygous/homozygous nonsense variant p. $\left(\mathrm{C} 107^{*}\right)$.

Assessment of dental development and determination of dental age in patients with oligodontia is impaired by the estimation of developmental stages for missing teeth, particularly in the case of oligodontia with a higher number of missing teeth. A specific method for assessing dental age in patients with oligodontia is not available, which may have prevented comparable investigations. Dental age was assessed in patients with cerebral palsy and Down syndrome, a risk group for oligodontia ${ }^{49}$ by applying two methods, the one described by Nolla ${ }^{50}$ and the other one described by Demirjian. ${ }^{44}$ The limitation of the method described by $\mathrm{Nolla}^{50}$ is the impossibility of applying this method in its original form because it tends to underestimate dental age. ${ }^{51,52}$ The limitation of the method described by Demirjian ${ }^{44}$ is the trend to overestimate dental age due to the acceleration of dental development. ${ }^{53,54}$ To address the limitations of the existing methods, a combined method was applied in the present study with regression equations for missing teeth in case no corresponding mandibular or maxillary tooth was available. ${ }^{45}$ An alternative could have been to skip the estimations from corresponding maxillary teeth and to apply regression equations directly in case a tooth was missing in both sides of the mandible, as the eruption of mandibular teeth precedes 
maxillary teeth by 1 year at most. This would be expressed in less than one stage of dental development and not necessarily contribute to a change of dental age. As the equations are based on a general population, however, it can be questioned whether these can be inferred to patients with oligodontia. For this reason, information from the same patient was preferred for this study.

In general, the complete development of a tooth takes at least 10 years. The findings of this study suggest $\sim 3$ years delay in dental development for patients with oligodontia and WNT10A variants. A previous study revealed an association between a decreased expression of WNT10A and inhibition of dentin apposition and root elongation. ${ }^{30}$ Furthermore, WNT10A defects can lead to molar crown and root dysmorphologies. ${ }^{31}$ The present study showed that the left maxillary canine and the left mandibular second molar had statistically significant shorter roots in patients with WNT10A variants compared with patients without WNT10A variants. This result was confirmed by the ordinal regression analysis, where development of the maxillary canine and second molars was 5-6 stages delayed in patients with WNT10A variants compared with patients without WNT10A variants. As in the literature, WNT10A variants are associated with both the type and number of missing teeth, ${ }^{36}$ we expected significant effect of WNT10A variants on development of more teeth. Even though no statistically significant result was obtained for the other present teeth, in general the effect estimates were negative showing a trend of shorter roots and lower stages of development for present teeth in patients with WNT10A variants.

WNT10A variants in this study were found in cases with more delayed dental development and more missing teeth (Figure 2). Another recent finding indicates that the association with WNT10A is stronger with an increasing number of missing teeth and presence of the nonsense variant c. $\left(321 \mathrm{C}>\mathrm{A}\right.$ p. $\left.\left(\mathrm{C} 107^{*}\right)\right) .{ }^{48}$ These arguments highlight that WNT10A is not only involved in tooth germ formation but has a role in the subsequent stages of tooth development as well. Further investigations on the effect of other genes are necessary for a better understanding of the relation between oligodontia and delayed dental development.

\section{CONFLICT OF INTEREST}

The authors declare no conflict of interest.

\section{ACKNOWLEDGEMENTS}

We thank colleagues in the Department of Medical Genetics and Oral and Maxillofacial Surgery, Prosthodontics and Special Dental Care in the University Medical Centre Utrecht for the referral of patients and molecular analysis performance.

1 Silva Meza R: Radiographic assessment of congenitally missing teeth in orthodontic patients. Int J Paediatr Dent 2003; 13: 112-116.

2 Goodman JR, Jones SP, Hobkirk JA, King PA: Hypodonta 1: clinical features and the management of mild to moderate hypodontia. Dent Update 1994; 21: 381-384.

3 Kotecha S, Turner PJ, Dietrich T, Dhopatkar A: The impact of tooth agenesis on oral health-related quality of life in children. J Orthod 2013; 40: 122-129.

4 Polder BJ, Van't Hof MA, Van der Linden FP, Kuijpers-Jagtman AM: A meta-analysis of the prevalence of dental agenesis of permanent teeth. Community Dent Oral Epidemiol 2004; 32: 217-226.

5 Shimizu T, Maeda T: Prevalence and genetic basis of tooth agenesis. Jpn Dent Sci Rev 2009; 45: 52-58.

6 Khalaf K, Miskelly J, Voge E, Macfarlane TV: Prevalence of hypodontia and associated factors: a systematic review and meta-analysis. J Orthod 2014; 41: 299-316.

7 van den Boogaard M-J, Créton M, Bronkhorst $Y$ et al: Mutations in WNT10A are present in more than half of isolated hypodontia cases. J Med Genet 2012; 49: 327-331.

8 Schalk-van der Weide Y: Oligodontia: A Clinical, Radiographic and Genetic Evaluation. Universiteit Utrecht: faculteit Geneeskunde, 1992; 7: 3-27.
9 Rushmah M: Hypodontia of the primary and permanent dentition. J Clin Pediatr Dent 1992; 16: 121-123.

10 Nieminen P: Genetic basis of tooth agenesis. J Exp Zool B Mol Dev Evol 2009; 312 : 320-342.

11 Weide YSV, Beemer FA, Faber JAJ, Bosman F: Symptomatology of patients with oligodontia. J Oral Rehabil 1994; 21: 247-261.

12 Creton M, Cune M, Verhoeven W, Muradin M, Wismeijer D, Meijer G: Implant treatment in patients with severe hypodontia: a retrospective evaluation. J Oral Maxillofac Surg 2010; 68: 530-538.

13 Wong AT, McMillan AS, McGrath C: Oral health-related quality of life and severe hypodontia. J Oral Rehabil 2006; 33: 869-873.

14 Schalk-van der Weide Y, Steen WH, Bosman F: Taurodontism and length of teeth in patients with oligodontia. J Oral Rehabil 1993; 20: 401-412.

15 Tunc ES, Bayrak S, Koyuturk AE: Dental development in children with mild-tomoderate hypodontia. Am J Orthod Dentofacial Orthop 2011; 139: 334-338.

16 Uslenghi S, Liversidge HM, Wong FS: A radiographic study of tooth development in hypodontia. Arch Oral Biol 2006; 51: 129-133.

17 Ruiz-Mealin EV, Parekh S, Jones SP, Moles DR, Gill DS: Radiographic study of delayed tooth development in patients with dental agenesis. Am J Orthod Dentofacial Orthop 2012; 141: 307-314.

18 Ben-Bassat Y, Babadzhanov D, Brin I, Hazan-Molina H, Aizenbud D: Maturation of teeth adjacent to dental agenesis site. Acta Odontol Scand 2014; 72: 516-522.

19 Brook AH, Elcock C, al-Sharood MH, Mckeown HF, Khalaf K, Smith RN: Further studies of a model for the etiology of anomalies of tooth number and size in humans. Connect Tissue Res 2002; 43: 289-295.

20 Kennedy DJ: Partial anodontia: a brief review and a case report of multiple familial incidence. Oral Surg Oral Med Oral Pathol 1950; 3: 63-73.

21 Vastardis $\mathrm{H}$ : The genetics of human tooth agenesis: new discoveries for understanding dental anomalies. Am J Orthodont Dentofacial Orthop 2000; 117: 650-656.

22 Kolenc-Fusé FJ: Tooth agenesis: in search of mutations behind failed dental development. Med Oral Patol Oral Cir Bucal 2003; 9: 390-395, 385-390.

23 Caton J, Tucker AS: Current knowledge of tooth development: patterning and mineralization of the murine dentition. J Anat 2009; 214: 502-515.

24 Cobourne MT, Sharpe PT: Making up the numbers: the molecular control of mammalian dental formula. Semin Cell Dev Biol 2010; 21: 314-324.

25 De Coster PJ, Marks LA, Martens LC, Huysseune A: Dental agenesis: genetic and clinical perspectives. J Oral Pathol Med 2009; 38: 1-17.

26 Mitsiadis TA, Smith MM: How do genes make teeth to order through development? J Exp Zool B Mol Dev Evol 2006; 306: 177-182.

27 Dhanrajani PJ: Hypodontia: etiology, clinical features, and management. Quintessence Int 2002; 33: 294-302.

28 Matalova E, Fleischmannova J, Sharpe PT, Tucker AS: Tooth agenesis: from molecular genetics to molecular dentistry. J Dent Res 2008; 87: 617-623.

29 Cobourne MT: Familial human hypodontia-is it all in the genes? Br Dent J 2007; 203: 203-208.

30 Bae CH, Kim TH, Ko SO, Lee JC, Yang X, Cho ES: Wntless regulates dentin apposition and root elongation in the mandibular molar. J Dent Res 2015; 94: 439-445.

31 Yang J, Wang SK, Choi $M$ et al: Taurodontism, variations in tooth number, and misshapened crowns in Wnt10a null mice and human kindreds. Mol Gene Genomic Med 2015; 3: 40-58.

32 Chen J, Lan Y, Baek JA, Gao Y, Jiang R: Wnt/beta-catenin signaling plays an essential role in activation of odontogenic mesenchyme during early tooth development. Dev Biol 2009; 334: 174-185.

33 Fujimori S, Novak $\mathrm{H}$, Weissenbock $\mathrm{M}$ et al: Wnt/beta-catenin signaling in the dental mesenchyme regulates incisor development by regulating Bmp4. Dev Biol 2010; 348: 97-106.

34 Massink MP, Creton MA, Spanevello F et al: Loss-of-function mutations in the WNT co-receptor LRP6 cause autosomal-dominant oligodontia. Am J Hum Genet 2015; 97: 621-626.

35 Arte S, Parmanen S, Pirinen S, Alaluusua S, Nieminen P: Candidate gene analysis of tooth agenesis identifies novel mutations in six genes and suggests significant role for WNT and EDA signaling and allele combinations. PLoS One 2013; 8: e73705.

36 Arzoo PS, Klar J, Bergendal B, Norderyd J, Dahl N: WNT10A mutations account for (1/4) of population-based isolated oligodontia and show phenotypic correlations. Am J Med Genet A 2014; 164A: 353-359.

37 Kantaputra P, Sripathomsawat W: WNT10A and isolated hypodontia. Am J Med Genet A 2011; 155: 1119-1122.

38 Adaimy L, Chouery E, Mégarbané $\mathrm{H}$ et al: Mutation in WNT10A is associated with an autosomal recessive ectodermal dysplasia: the odonto-onycho-dermal dysplasia. Am J Hum Genet 2007; 81: 821-828.

39 Nawaz S, Klar J, Wajid M et al: WNT10A missense mutation associated with a complete odonto-onycho-dermal dysplasia syndrome. Eur J Hum Genet 2009; 17: 1600-1605.

40 Bohring A, Stamm T, Spaich C et al: WNT10A mutations are a frequent cause of a broad spectrum of ectodermal dysplasias with sex-biased manifestation pattern in heterozygotes. Am J Hum Genet 2009; 85: 97-105.

41 Cluzeau C, Hadj-Rabia S, Jambou M et al: Only four genes (EDA1, EDAR, EDARADD, and WNT10A) account for $90 \%$ of hypohidrotic/anhidrotic ectodermal dysplasia cases. Hum Mutat 2011; 32: 70-72.

42 Van Wijk AJ, Tan SPK: A numeric code for identifying patterns of human tooth agenesis: a new approach. Eur J Oral Sci 2006; 114: 97-101.

43 Creton MA, Cune MS, Verhoeven W, Meijer GJ: Patterns of missing teeth in a population of oligodontia patients. Int J Prosthodont 2007; 20: 409-413. 
44 Demirjian A, Goldstein H, Tanner JM: A new system of dental age assessment. Hum Biol 1973; 211-227.

45 Nyström M, Aine L, Peck L, Haavikko K, Kataja M: Dental maturity in Finns and the problem of missing teeth. Acta Odontologica 2000; 58: 49-56.

46 Leurs IH, Wattel E, Aartman IHA, Etty E, Prahl-Andersen B: Dental age in Dutch children. Eur J Orthod 2005; 27: 309-314.

47 Nordgarden H, Jensen JL, Storhaug K: Oligodontia is associated with extraoral ectodermal symptoms and low whole salivary flow rates. Oral Dis 2001; 7: 226-232.

48 Song S, Zhao R, He H, Zhang J, Feng H, Lin L: WNT10A variants are associated with non-syndromic tooth agenesis in the general population. Hum Genet 2014; 133 : 117-124.
49 Russell BG, Kjær I: Tooth agenesis in Down syndrome. Am J Med Genet 1995; 55 : 466-471.

50 Nolla CM: The development of the permanent teeth. J Dent Child 1960; 27: 254-266.

51 Briffa K, Busuttil Dougall N, Galea J, Mifsud D, Camilleri S: Chronologic and dental ages of Maltese schoolchildren: a pilot study. MMJ 2005; 33: 36-40.

52 Kumaresan R, Cugati N, Chandrasekaran B, Karthikeyan P: Reliability and validity of five radiographic dental-age estimation methods in a population of Malaysian children. J Investig Clin Dent 2014; 7: 102-109.

53 Nadler GL: Earlier dental maturation: fact or fiction? Angle Orthod 1998; 68 535-538.

54 Holtgrave EA, Kretschmer R, Müller R: Acceleration in dental development: fact or fiction. Eur J Orthod 1997; 19: 703-710.

Supplementary Information accompanies this paper on European Journal of Human Genetics website (http://www.nature.com/ejhg) 